\title{
TIMING ACQUISITION FOR FRACTAL MODULATION IN GAUSSIAN WHITE AND $1 / F$ CHANNELS
}

\author{
$\dagger$ Chih-Ming Fu, $\ddagger$ Wen-Liang Hwang and $\dagger$ Chung-Lin Huang \\ $\dagger$ Department of Electrical Engineering, National Tsing Hua University, Taiwan \\ $\ddagger$ Institute of Information Science, Academia Sinica, Taiwan
}

\begin{abstract}
We propose a clock acquisition algorithm for timing recovery for fractal modulation in additive white Gaussian noise (AWGN) and $1 / f$ channels. Our acquisition algorithm uses exclusively the data redundancy inherently built in the fractal modulation to locate the beginning of timing of all the subbands simultaneously. Different diversity techniques can then be applied after the beginning of the data block is obtained. Our acquisition functions are derived by using the maximum-likelihood method. Using a serial search algorithm to maximize the acquisition function, we may find the timing point. Simulations have been conducted in evaluating the mean acquisition time of our algorithm.
\end{abstract}

Keywords: Fractals, Synchronization, Wavelet transforms.

\section{INTRODUCTION}

In a communication scenario, a channel is opened for a duration of time to a receiver which takes a certain bandwidth during that period. The wavelet-based fractal modulation method was proposed for this case where the data are transmitting in a channel whose duration and bandwidth are simultaneously unknown to the transmitter [1]. Analysis results of the impact of the clock error on BER performance due to the data diversity for fractal modulation in an AWGN channel are also investigated [2]. We will propose clock acquisition methods for fractal modulated self-similar signals embedded in various kinds of noises. We assume that signals are transmitted in baseband and that the time domain clock error is the major factor that degrades the performance of a receiver.

We derive our acquisition function for fractal modulation by using the maximum-likelihood method in AWGN and $1 / f$ channels. This function makes use of the information diversity in time frequency cells in locating the beginning clock of all the subbands. The acquisition function is bursting just as the code acquisition function in the CDMA system. Therefore, we use a serial search algorithm such as that used in CDMA code acquisition for the maximumlikelihood solutions of our acquisition function[3]. The pa- rameters related to the performances in terms of the mean acquisition time of our acquisition algorithms have been discussed and the simulation results on the performance are given.

This paper is organized as follows. In Section 2, we firstly review the wavelet transform and the fractal modulation. Then we introduce the modulation and demodulation fractal bases. These bases are weighted linear combinations of the dyadic wavelet bases whose corresponding time-frequency cells contain the same data in a fractal modulated signal. In Section 3, We also derive the clock acquisition functions by the maximum-likelihood methods in an AWGN channel and in a $1 / f$ channel. A serial search algorithm is introduced in Section 3.2 to find the location that maximizes the acquisition function. The simulation results on the acquisition performance are shown in Section 4. Finally, our conclusion is given in the last section.

\section{FRACTAL BASES FOR MODULATION AND DEMODULATION}

We use a fractal group as a convenient notation to refer to a collection of time-scale indices taking the same information $d_{(n \bmod L)}$ in a time frequency portrait. If the data length is $L$, then we will have a total of $L$ fractal groups. The time-scale indices belonging to the fractal group $G_{i}$ are $\{(m, n) \mid m=0,1, \ldots, M-1$ and $(n \bmod L)=i\}$, where $m$ and $n$ are scale and time indices, respectively. A fractal modulation takes a discrete sequence $\left\{d_{n}\right\}$ to a self-similar signal by performing an inverse wavelet transform

$$
s(t)=\sum_{i=0}^{L-1} d_{i} \sum_{(m, n) \in G_{i}} \beta^{-m / 2} \psi_{m, n}(t)=\sum_{i=0}^{L-1} d_{i} \varphi_{i}(t),
$$

where

$$
\varphi_{i}(t)=\sum_{(m, n) \in G_{i}} \beta^{-m / 2} \psi_{m, n}(t)
$$

is a weighted summation of wavelets with time-scale indices containing $d_{i}$ in fractal modulation. In the demodulation, 
we use the other basis. The demodulation waveform (also called demodulation fractal basis) $\bar{\varphi}_{i}(t)$ is defined as

$$
\bar{\varphi}_{i}(t)=\sum_{(m, n) \in G_{i}} \beta^{m / 2} \psi_{m, n}(t) .
$$

The modulation and demodulation fractal bases are a linear combination of the wavelet basis but with a different weighting function. They are self-similar and satisfy the mutually orthogonal property:

$$
\begin{aligned}
& <\varphi_{i}(t) \bar{\varphi}_{k}(t) d t>=\eta_{i} \delta_{i, k} \\
& <\varphi_{i}(t) \varphi_{k}(t) d t>=\eta_{i}^{m} \delta_{i, k} \\
& <\bar{\varphi}_{i}(t) \bar{\varphi}_{k}(t) d t>=\eta_{i}^{d} \delta_{i, k}
\end{aligned}
$$

where $\eta_{i}, \eta_{i}^{d}$ and $\eta_{i}^{m}$ are defined as,$\sum_{(m, n) \in G_{i}} 1$, and $\sum_{(m, n) \in G_{i}} \beta^{m}, \sum_{(m, n) \in G_{i}} \beta^{-m}$ respectively. We hereafter consider a convenient case when all fractal groups has the same number of elements. In such a case, if there are $M$ subbands, then we have $\eta=\eta_{i}=2^{M}-1, \eta_{m}=\eta_{i}^{m}=$ $\frac{1-\left(2 \beta^{-1}\right)^{M}}{1-2 \beta^{-1}}$, and $\eta_{d}=\eta_{i}^{d}=\frac{1-(2 \beta)^{M}}{1-2 \beta}$. The $\eta$ will be called the redundancy factor.

\section{TIMING ACQUISITION}

Fractal modulation diversifies a bit into many wavelet time frequency cells. We take advantage of this diversity by demodulating all the cells with the same bit information together and acquire a correct timing of the beginning of all subbands. A received waveform with transmission delay $\tau$ can be written as $r(t)=s(t-\tau)+w(t)$. A derivation of the maximum-likelihood solution for block time acquisition when a signal is embedded in AWGN is given as follows:

$$
\begin{aligned}
& r_{i}(\hat{\theta})=<r(t), \bar{\varphi}_{i}(t-\hat{\tau})> \\
& s_{i}(\hat{\theta})=<s(t-\tau), \bar{\varphi}_{i}(t-\hat{\tau})> \\
& \frac{1}{T_{0}} \int_{T_{o b s}}[r(t)-s(t-\hat{\tau})]^{2} d t=\frac{\eta_{m}}{\eta^{2}} \sum_{i=0}^{L-1}\left(r_{i}(\hat{\theta})-s_{i}(\hat{\theta})\right)^{2} .
\end{aligned}
$$

where $\hat{\theta}=(\hat{\tau}-\tau)$ is normalized clock error. The maximum likelihood method finds the $\hat{\tau}^{*}$ that maximizes the likelihood function. We reformulate the above equation and obtain

$$
\Lambda_{L}(\hat{\theta})=\eta_{m} \eta \sum_{i} d_{i} r_{i}(\hat{\theta}),
$$

where the log-likelihood function depends on modulation and demodulation fractal bases, the information vector, and the noise added to the signal. Note that the data $d_{i}$ for $i=0 \cdots L-1$ are required in computing the location that maximizes the log-likelihood function. Here, we assume that the data are obtained by using a decision direct technique or a training sequence. We then use $\hat{d}_{i}$ to denote the estimated $d_{i}$ and by a proper normalization such that the maximum value becomes 1 to obtain the normalized loglikelihood function:

$$
\Lambda_{a c q}(\hat{\theta})=\frac{1}{\eta} \sum_{i} \hat{d}_{i} r_{i}(\hat{\theta})
$$

where $\hat{d}_{i}$ is the estimation of $d_{i}$. Since $\Lambda_{a c q}(\hat{\theta})$ is a bursty function (an example is shown in Fig.1) we cannot locate the maximum value position of this function by taking the derivative and updating the information required for timing control loop. A serial state search algorithm will be introduced in Section 3.2 to find the maximum value timing point of $\Lambda_{a c q}(\hat{\theta})$.
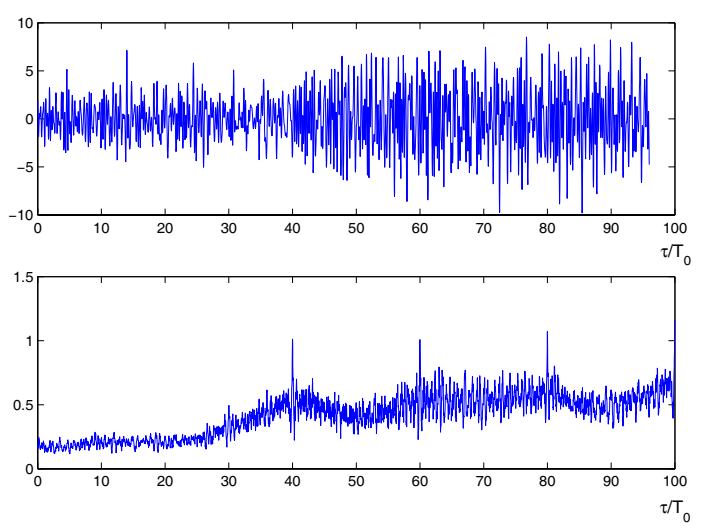

Fig. 1. Top: The received noisy signal. The transmitted selfsimilar signal contains 3 data blocks and each block has 20 symbols. Bottom: The normalized log-likelihood function is a function of normalized clock error. There are 3 peaks and each peak appears in every $20 \tau / T_{0}$.

\subsection{Spectrum Matching - Timing Acquisition in a $1 / f$ noise}

When a receiver is operating in a channel with a known bandwidth, the appropriate spectrum shaping of the transmitted signal is governed by a "water-filling" procedure in which more signal power is distributed to the frequency where the noise power is smaller. When there is an uncertainty in the available bandwidth of a receiver, the waterfilling approach may lead to poor worst-case performance since it is possible that most of the signal power is allocated to the frequencies that are not used by the receiver. Thus, the "water-filling" loading algorithm is not well-suited for fractal modulation since a receiver can operate with any amount of bandwidth. Contrast to the "water-filling" procedure, the signal power may be distributed proportionally to the noise spectrum so that a "flat" $S N R$ is obtained independent of 
any frequency. This "spectrum matching" rule maintains the $S N R$ across all the frequencies and leads to a uniform performance for a receiver operating with varying bandwidth. This rule is potentially well-suited for transmitting fractal modulated signals.

We shall discuss fractal modulation and demodulation of a noisy signal which is embedded in a $1 / f$ noise using a spectrum matching approach. A $1 / f$ is a self-similar stochastic process. It appears in many physical phenomena and is also frequently used in modeling errors on communication channels. The process is characterized by a fractal parameter of degree $H$. If $H=1 / 2$, then it is the Brownian motion process. Many properties of a $1 / f$ process have been thoroughly discussed. Also, wavelet-based algorithms have been proposed to estimate the fractal parameter robustly and efficiently .

We assume that the noise $w(t)$ is a Gaussian $1 / f$ process whose degree $H_{w}=H$ has been estimated with a separate procedure, and that the degree $H_{s}$ of the signal $s(t)$ has been chosen to make it 'look like' the transmitted noise such that

$$
H_{s}=H_{w}=H
$$

It was shown that an orthogonal wavelet is an almost whitening filter of any $1 / f$ process [4] and the wavelet coefficients of a Gaussian $1 / f$ process can be well approximated as independent zero-mean Gaussian random variables with variance depending on the scale and the fractal parameter $H$. we obtain

$p(\mathbf{r} \mid \hat{\theta})=\left(\frac{1}{\sqrt{2 \pi} \sigma_{m}}\right)^{L} \exp \left\{-\sum_{i=0}^{L-1} \frac{\left[r_{i}(\hat{\theta})-s_{i}(\hat{\theta})\right]^{2}}{2 \sigma_{m}^{2}}\right\}$.

Therefore, we can use the same log-likelihood equation in the case of AWGN (see Eq.(1)) for timing acquisition.

\subsection{Serial Search Algorithm}

The parameter of an acquisition algorithm is the mean acquisition time to search for a clock timing sufficiently close to the beginning of the data block. This is essentially an estimation problem and many solutions have been proposed. When a log-likelihood function is bursty, such as a code acquisition function for CDMA, so that the location of its maximum value cannot be identified easily from its derivative by a gradient decent approach, a well-known and simple acquisition method is the serial search algorithm [3].

Fig. 2 shows a diagram of the serial search algorithm. There is a sequence of $\nu$ points in the outer circle to be searched. The accuracy of the acquired clock to within less than $T_{c}$ of the real synchronization point depends on $\nu$. The label in a branch gives the probability that a transition occurs between points, multiplied by a power of $z$, where $P_{D}$ and $P_{F}$ respectively indicate the detection probability and the false alarm probability in testing whether the point is the correct timing hypothesis. If the power of $z$ is $n$, then it indicates that $n \tau_{D}$ seconds required to make the transition. If a true hit is observed, as with the branch labeled $P_{D} z$, then the system has acquired the timing and the search is completed. Otherwise, a penalty of obtaining a false alarm to the value of $k \tau_{D}$ seconds, as with the branch labeled $z^{K}$, is paid, and then the system moves to the next point in the outer circle. This procedure will be repeated until the timing is finally acquired correctly. The search time is the sum of the transition times of all the branches on the path taken in the diagram with the assumption that any point is equally likely to be the initial point of the path. The serial search algorithm parameters are given in the next section. The mean acquisi-

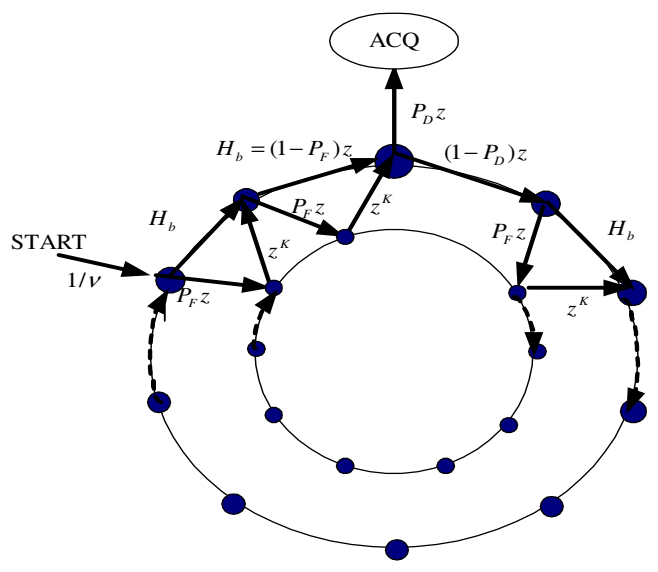

Fig. 2. A serial search state diagram. The points in the inner circle are false alarm states.

tion time for evaluating our acquisition algorithm have been derived. Here, we only sketch their derivations for convenience. Let us call the point when the acquisition timing is correctly acquired the destination point. Observe that any branch into the inner circle returns to the next outer circle point with a delay of $K+1$. Thus, the transition from an outer circle point, other than the destination point, to the next outer circle point has the branch transfer function

$$
H_{b}(z)=\left(1-P_{F}\right) z-P_{F} z^{K+1} .
$$

For the usual case when $\nu \gg 1$, the mean acquisition time can in terms of $P_{F}$ and $P_{D}$ approximately be as follows:

$$
T_{a c q} \cong \frac{\left(2-P_{D}\right)\left(1+K P_{F}\right)}{2 P_{D}}\left(\nu \tau_{D}\right) .
$$

Suppose that $\nu \gg 1$, the variance can be written in terms of $P_{D}$ and $P_{F}$ :

$$
\begin{aligned}
& \tau_{D}^{2}\left\{\left(1+K P_{F}\right)^{2} \nu^{2}\left(\frac{1}{12}-\frac{1}{P_{D}}+\frac{1}{P_{D}^{2}}\right)\right. \\
& +6 \nu\left[K(K+1) P_{F}\left(2 P_{D}-P_{D}^{2}\right)\right. \\
& \left.\left.+\left(1+P_{F} K\right)\left(4-2 P_{D}-P_{D}^{2}\right)\right]+\frac{1-P_{D}}{P_{D}^{2}}\right\} .
\end{aligned}
$$




\section{SIMULATION RESULTS}

Various experiments have been performed to evaluate the performance of our clock acquisition algorithm for fractal modulation. All the simulation programs are written in Matlab and using Meyer wavelet. Our wavelets $\psi_{m, n}(t)$ are approximated by discrete points. We use 128 points for the signaling interval $T_{0}$ at the scale $m=0$. In all the experiments, we use the Meyer wavelet which has a length of $16 T_{0}$ at that scale. Thus, the Meyer wavelet $\psi(t)$ is approximated by 2048 discrete points.

The serial search algorithm parameters used here are selected as follows (assuming no Doppler): Points in the outer circle are sampled at every one-half of the fast subband symbol timing. Thus, if we have $M$ subbands and a data block with $L$ symbols and each symbol is either $\sqrt{E_{b}}$ or $-\sqrt{E_{b}}$, then we will have $L 2^{M-1}$ symbols in the fastest subband. Hence, there are $\nu=L 2^{M}$ points in the outer circle. The accuracy of our acquisition algorithm to the beginning of data block, $T_{c}$, is within one-half of the fastest subband. The correct timing hypothesis is tested at any point in the outer circle by comparing the $\Lambda_{a c q}(\tau)$ at that point to a given threshold and the hypothesis has passed if the $\Lambda_{a c q}(\tau)$ at that point is greater than the threshold. The maximum value of $\Lambda_{a c q}(\tau)$ is 1 . A low threshold results in an increase of the false alarm probability while a high threshold will decrease the detection probability. In both cases, the mean acquisition time increases. In all the experiments, our threshold is set to 0.7 , which may not be the optimal threshold for minimizing the mean acquisition time. The detection probability, $P_{D}$, and the false alarm probabilities, $P_{F}$, used in estimating the mean acquisition time were obtained through simulations. Like in [5], the $K$ is set to 7 .

\section{CONCLUSION}

We propose the clock timing acquisition algorithm which uses a serial search algorithm, similar to that used in CDMA for code acquisition, in locating the beginning timing of all the subbands in a delay signal. The acquisition algorithm obtains the maximum-likelihood solution in AWGN and $1 / f$ channels. We can then use the beginning of the data block to realize applications that adopt different diversity techniques. We show analytical results of the data redundancy (in terms of the redundancy factor $\eta$ ) and the performance in an AWGN channel for fractal modulation. The larger $\eta$ is, the better performance will be.
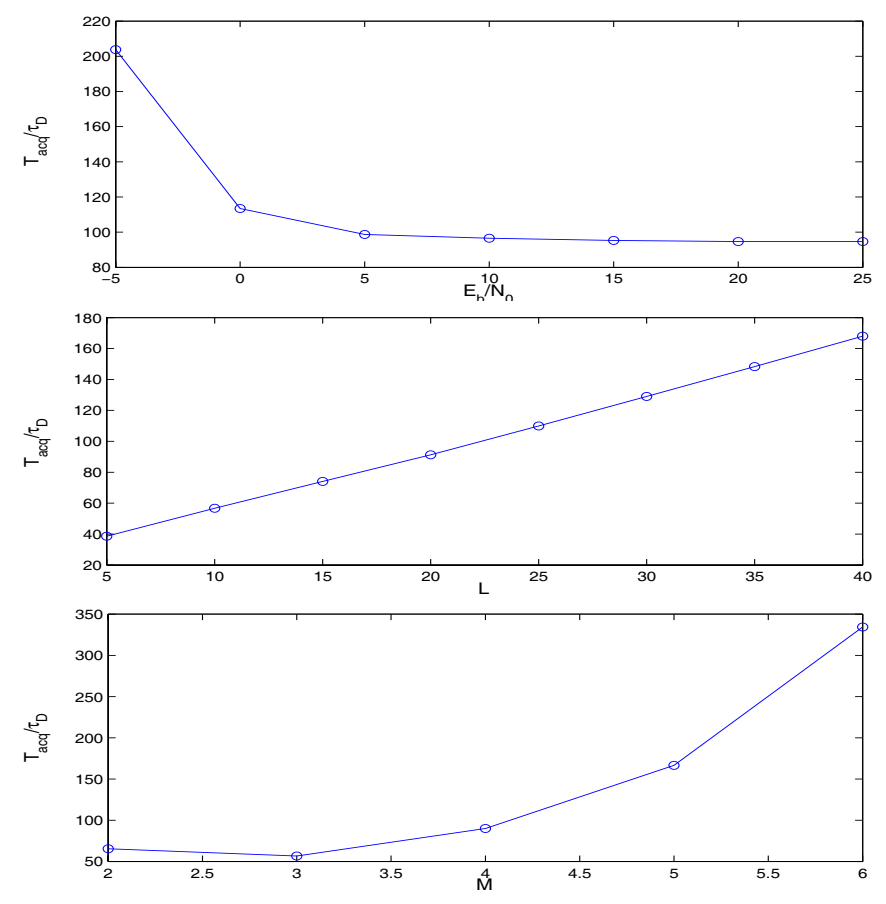

Fig. 3. Top:The normalized mean acquisition time with different $E_{b} / N_{0}$ in an AWGN channel. Experimental parameters are: $L=10, M=3$, and $H=-\frac{1}{2}$. The $E_{b} / N_{0}$ is measured at the slowest subband. Middle: Parameter is data block length $L$. Other parameters are: $M=3, H=-\frac{1}{2}$, and $E_{b} / N_{0}=0 \mathrm{~dB}$ which is measured at the slowest subband. Bottom: Parameter is numbers of subbands $M$. Other parameters are: $L=10, H=-\frac{1}{2}$, and $E_{b} / N_{0}$ at the slowest subband is $0 \mathrm{~dB}$.

\section{REFERENCES}

[1] G. Wornell and A. V. Oppenheim, "Wavelet based representations for a class of self similar signals with applications to fractal modulation," IEEE Trans. on Inform. Theory, vol. 38, pp. 785-800, May 1992.

[2] C. M. Fu, W. L. Hwang, and C. L. Huang, "Clock synchronization for fractal modulation," IEEE ICASSP, pp. 2810-2812, 2002.

[3] A. J. Viterbi, Principles of Spread Spectrum Communicaion, ADDISON-WESLEY Press, 1995.

[4] G. Wornell, "A karhunen-loeve expansion for 1/f processes via wavelets," IEEE Trans. on Information Theory, vol. 36, pp. 859-861, July 1990.

[5] L. L. Yang and L. Hanzo, "Serial acquisition of DSCDMA signals in multipath fading mobile channels," IEEE Transaction on vehicular technology, vol. 50, pp. 278-286, March 2001. 